TRANSACTIONS OF THE

AMERICAN MATHEMATICAL SOCIETY

Volume 351, Number 2, February 1999, Pages 569-580

$\mathrm{S} 0002-9947(99) 02207-2$

\title{
WINDOWS OF GIVEN AREA WITH MINIMAL HEAT DIFFUSION
}

\author{
JOCHEN DENZLER
}

\begin{abstract}
For a bounded Lipschitz domain $\Omega$, we show the existence of a measurable set $D \subset \partial \Omega$ of given area such that the first eigenvalue of the Laplacian with Dirichlet conditions on $D$ and Neumann conditions on $\partial \Omega \backslash D$ becomes minimal. If $\Omega$ is a ball, $D$ will be a spherical cap.
\end{abstract}

\section{INTRODUCTION}

We are considering the lowest eigenvalue $\lambda_{1}$ of the Laplacian in a bounded Lipschitz domain $\Omega$. The boundary conditions are Dirichlet on one part $D$ of $\partial \Omega$ and Neumann elsewhere:

$$
\lambda_{1}(D):=\inf \left\{\int_{\Omega}|\nabla u|^{2} d x\left|u \in H^{1}(\Omega), \int_{\Omega} u^{2}=1, u\right|_{D}=0\right\},
$$

where $H^{1}$ denotes the Sobolev space of functions with square integrable first derivatives.

This eigenvalue can be interpreted as the heat diffusion rate through a noninsulated window in an otherwise perfectly insulated room, asymptotically for large time; an interpretation which should not be taken too literally, because no convection is taken into account. However, considering $\lambda_{1}$ as a function of the "window" $D$ is the source for a lot of intriguing geometric inverse spectral problems of a type that has apparently not been considered so far. One key problem is to minimize $\lambda_{1}$ under the constraint of fixed area $\mu(D)$ of $D$. It is the main purpose of this paper to show that such a minimum (we call it optimal window) actually exists.

Mixed boundary data are by their nature so severely discontinuous from the elliptic regularity point of view that smoothness of $\partial \Omega$ would be an assumption quite alien to the nature of the problem. Bearing with this opinion makes it necessary to employ recent tools on elliptic problems with minimal regularity as described in [8]. While the author cannot pretend to contribute to this theory, he hopes that this application might contribute to popularizing the results of the theory. The last section contains the proof, kindly supplied by C. Kenig, for the theorem that is needed to carry the existence proof over to nonsmooth boundary. The responsibility for any insufficiency in the exposition of this proof remains with the author. For smooth boundary, the existence proof can also be carried through with more classical tools.

Received by the editors November 16, 1996.

1991 Mathematics Subject Classification. Primary 49J40; Secondary 49J10, 35J20, 35R05.

(C)1999 American Mathematical Society 
Whereas the shape of optimal windows is not exhibited by our existence proof and leaves open many questions, symmetrization arguments give a complete solution when $\Omega$ is a ball. Some heuristic discussion of the shape question as well as quantitative lower bounds for the eigenvalue have been given by the author in [4]. Moreover, it has been shown there that it is not possible to maximize $\lambda_{1}$ under the area constraint.

\section{Existence of Optimal Windows}

In view of $(0.1)$, to finding a minimizer for the variational problem

$$
\ell(A):=\inf \left\{\lambda_{1}(D) \mid \mu(D)=A\right\}
$$

is equivalent to finding a minimizer of

$$
\ell(A)=\inf \left\{I[u, \chi]:=\int_{\Omega}|\nabla u|^{2} \mid(u, \chi) \in \mathcal{D}(\Omega, A)\right\},
$$

where

$$
\begin{aligned}
\mathcal{D}(\Omega, A):= & \left\{(u, \chi) \in H^{1}(\Omega) \times L^{\infty}(\partial \Omega) \mid\right. \\
& \left.\int_{\Omega} u^{2}=1, \chi^{2}=\chi, \int_{\partial \Omega} \chi=A, u \cdot \chi=0 \in L^{2}(\partial \Omega)\right\}
\end{aligned}
$$

and the $u$ in $u \cdot \chi$ denotes the trace of $u \in H^{1}(\Omega)$ on the boundary $\partial \Omega$. (According to [1, 5.22], the restriction map $C^{0}(\bar{\Omega}) \rightarrow C^{0}(\partial \Omega)$ extends to a map in particular defined from $H^{1}(\Omega)$ to $L^{2}(\partial \Omega)$ and called the trace map.) Obviously, $\chi^{2}=\chi$ amounts to $\chi$ being a characteristic function of some set $D$; as $D$ is varying, we prefer to include the spurious dependence on $\chi=\chi_{D}$ into $I$. We first minimize the same functional in the larger class

$$
\begin{aligned}
\mathcal{E}(\Omega, A):= & \left\{(u, \phi) \in H^{1}(\Omega) \times L^{\infty}(\partial \Omega) \mid\right. \\
& \left.\int_{\Omega} u^{2}=1, \phi \leq 1, \int_{\partial \Omega} \phi \geq A, u \cdot \phi=0 \in L^{2}(\partial \Omega)\right\},
\end{aligned}
$$

which has more convenient functional analytic properties, and show

Theorem 1. For a bounded Lipschitz domain $\Omega$ and positive $A<\mu(\partial \Omega)$, there exists $(u, \phi) \in \mathcal{D}(\Omega, A)$ minimizing $I[u, \phi]$ in $\mathcal{E}(\Omega, A)$. Moreover, all minimizers of $I$ in $\mathcal{E}(\Omega, A)$ actually lie in $\mathcal{D}(\Omega, A)$.

To prove the theorem, let $\left\{\left(u_{n}, \phi_{n}\right)\right\}$ be a minimizing sequence, let $v_{n}:=\left|u_{n}\right|$ and let $\chi_{n}:=\chi_{\operatorname{supp} \phi_{n}}$ be the characteristic function of the support ${ }^{1}$ of $\phi_{n}$. As $\phi_{n} \leq 1$, we have $\chi_{n} \geq \phi_{n}$, and $\left(v_{n}, \chi_{n}\right)$ is also a minimizing sequence. By Hilbert space theory, Rellich's imbedding theorem, the compact trace map $H^{1}(\Omega) \rightarrow L^{2}(\partial \Omega)$ (see e.g. $[1$, thm. 5.4]) and passing to a subsequence, we get the following convergence results:

$v_{n} \rightarrow: v\left(\mathrm{w}-H^{1}(\Omega)\right), \quad v_{n} \rightarrow v\left(\mathrm{~s}-L^{2}(\Omega)\right.$ and s- $\left.L^{2}(\partial \Omega)\right), \quad \chi_{n} \rightarrow \phi\left(\mathrm{w}-L^{2}(\partial \Omega)\right)$.

We have the usual lower semi-continuity of the functional $I$, and under the above limits we salvage the following constraints: $0 \leq \phi \leq 1, \int_{\partial \Omega} \phi \geq A, v \geq 0, \int_{\Omega} v^{2}=1$,

\footnotetext{
${ }^{1}$ Here, the support of any $\phi \in L^{1}(\partial \Omega)$ is the set of all points, where $\phi$ doesn't vanish. It is only defined modulo null sets, which is enough to make $\chi_{\operatorname{supp} \phi}$ a well-defined element of $L^{\infty}(\partial \Omega)$. No operation of closure is involved.
} 
and $\int_{\partial \Omega} v \cdot \phi=0$. The latter implies $v \cdot \phi=0$ since $v, \phi \geq 0$. So we have found a minimizer $(v, \phi) \in \mathcal{E}(\Omega, A)$.

Letting $\chi:=\chi_{\operatorname{supp} \phi} \geq \phi$, we get another minimizer $(v, \chi)$, and it holds that $v \geq 0$ and $\chi^{2}=\chi$ for this one. We claim that actually $\int_{\partial \Omega} \chi=A$. This will already tell us that the particular minimizer $(v, \chi)$ lies indeed in the smaller class $\mathcal{D}(\Omega, A)$.

As

$$
A_{1} \leq A_{2} \Longrightarrow\left\{u \mid \exists \chi:(u, \chi) \in \mathcal{D}\left(\Omega, A_{1}\right)\right\} \supset\left\{u \mid \exists \chi:(u, \chi) \in \mathcal{D}\left(\Omega, A_{2}\right)\right\},
$$

it is clear that $A \mapsto \ell(A)$ is monotonic nondecreasing. So we can modify (1.1) to

$$
\ell(A)=\inf \left\{\lambda_{1}(D) \mid \mu(D) \geq A\right\}=\min \left\{\lambda_{1}(D) \mid \mu(D) \geq A\right\} .
$$

We claim that $\int_{\partial \Omega} \chi=A$ follows immediately from showing that $\ell$ is actually strictly monotonic. (See Figure 1.)

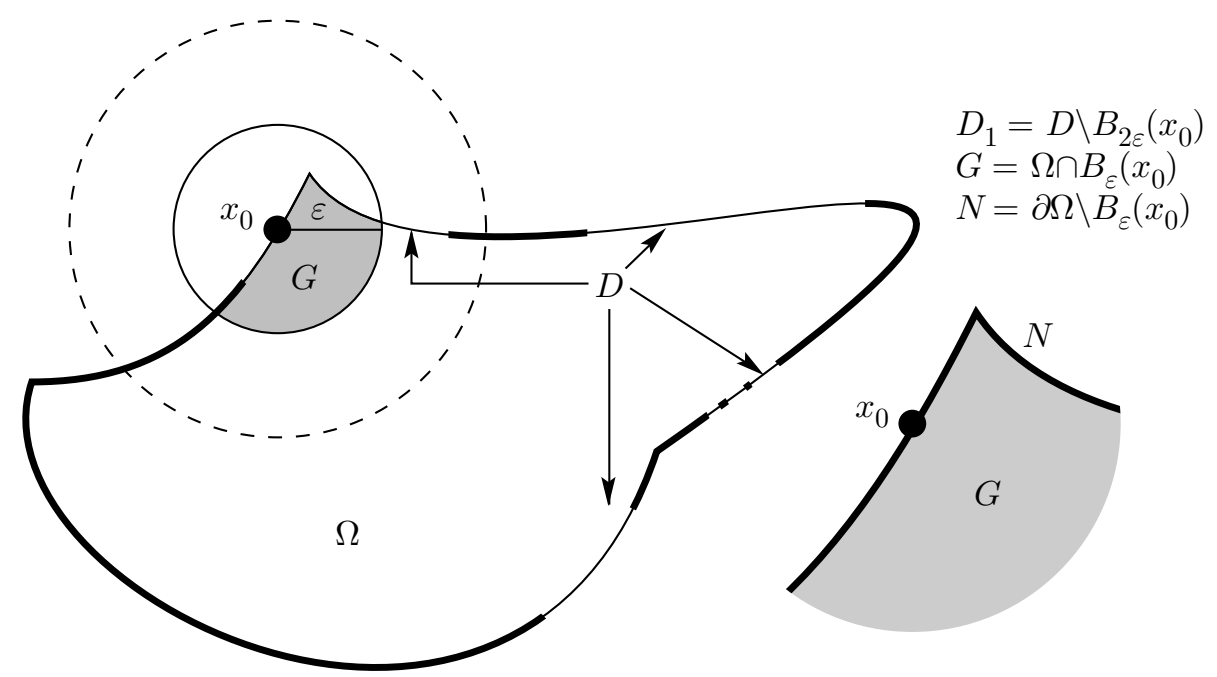

Figure 1. Geometric objects employed in the proof of strict monotonicity of $\ell(A)$

So suppose that we have a minimizer $(u, \chi)$ for $(1.5)$ with $\mu(\operatorname{supp} \chi)=\int_{\partial \Omega} \chi=$ $A_{2}$ and $D:=\operatorname{supp} \chi$. Let $A_{0}<A_{2}$ and choose $\varepsilon>0$ small enough such that any ball $B_{2 \varepsilon}\left(x_{0}\right)$ of radius $2 \varepsilon$ centered in some $x_{0} \in \partial \Omega$ intersects $\partial \Omega$ in a set of measure (on the boundary) less than $A_{2}-A_{0}$, and also small enough such that every $B_{2 \varepsilon}\left(x_{0}\right)$ lies entirely in one of some finite set of coordinate charts covering the boundary subject to the condition that in each chart, the domain can be described as the epigraph of a Lipschitz function. Choose $x_{0}$ such that $B_{\varepsilon}\left(x_{0}\right)$ intersects $D$ in a set of positive measure. Let $D_{1}:=D \backslash\left(B_{2 \varepsilon}\left(x_{0}\right) \cap \partial \Omega\right)$, and let its area be $A_{1}$. Then, $A_{0}<A_{1}<A_{2}$. Let $u_{1}$ be a minimizer for $(0.1)$ with the set $D_{1}$. This minimizer will be (Hölder) continuous up to the part $B_{\varepsilon}\left(x_{0}\right) \cap \partial \Omega$ of the boundary: [10], sections $3.13-14,[17]$. (We repeat some details of that argument in section 3.)

We will argue that $u_{1}$ cannot vanish anywhere on $B_{\varepsilon}\left(x_{0}\right) \cap \partial \Omega$ (or at least cannot vanish in a set of positive measure there). In contrast, $u$ does vanish in a set of positive measure in that part of the boundary (namely in $D$ ). Hence $u$ is not equal 
to the unique nonnegative minimizer $u_{1}$ for $(0.1)$ with $D_{1}$, and therefore

$$
\ell\left(A_{0}\right) \leq \ell\left(A_{1}\right) \leq \lambda_{1}\left(D_{1}\right)=\int\left|\nabla u_{1}\right|^{2}<\int|\nabla u|^{2}=\ell\left(A_{2}\right) .
$$

This finishes the strict monotonicity of $\ell$, and thus the existence of a minimizer in $\mathcal{D}(\Omega, A)$, subject to the claim that $u_{1} \neq 0$ in $B_{\varepsilon}\left(x_{0}\right) \cap \partial \Omega$.

We first establish this latter claim in the case of sufficiently smooth boundary (say $C^{2, \alpha}$ according to [2]). In this case, $u_{1}$ satisfies the classical homogeneous Neumann conditions. But Hopf's boundary point lemma [6, lemma 3.4], which needs to assume an interior sphere condition (which we have anyway for the $C^{2, \alpha}$ boundary), would imply a strictly negative outer normal derivative in any boundary point where $u_{1}$ vanishes.

If we actually need to deal with Lipschitz boundary, we cannot use the Hopf boundary point lemma, and so the above reasoning should be redone in a weak setting. The necessary tools are available and due to C. Kenig:

Theorem 2 (C. Kenig). Suppose $\Delta u \leq 0, u \geq 0$ in a bounded Lipschitz domain $\Omega$ in $\mathbb{R}^{d}$. Then, if there exists a subset $\Sigma$ of $\partial \Omega$ of positive measure on which both $u$ and its normal derivative vanish (in the sense of nontangential limits), then $u \equiv 0$ in $\Omega$.

This theorem, applied to $u_{1}$ on $G:=B_{\varepsilon}\left(x_{0}\right) \cap \Omega$ instead of $\Omega$, can replace the argument with boundary regularity and Hopf's boundary point principle in the above argument, therefore in the case of Lipschitz boundary, we still conclude that $u \neq u_{1}$.

In section 3 , we discuss why and in what sense $\partial_{n} u_{1}$ vanishes on $N:=\partial \Omega \cap$ $B_{\varepsilon}\left(x_{0}\right)$. Then we supply Kenig's proof for theorem 2 . This will conclude the proof of strict monotonicity of $\ell$ in the Lipschitz case and therefore the existence of a minimum in $\mathcal{D}(\Omega, A)$.

We still need to show that any minimizer $(u, \phi) \in \mathcal{E}(\Omega, A)$ is actually in $\mathcal{D}(\Omega, A)$. But the same argument as before can be repeated. We get $u \geq 0$ (or $u \leq 0$ ) automatically instead of enforcing it, because the (other) minimizer $|u|$ cannot vanish inside $\Omega$ due to the maximum principle. The inequalities

$$
\chi:=\chi_{\operatorname{supp} \phi} \geq \phi \quad \text { and } \quad A=\int \chi \geq \int \phi \geq A
$$

show that $\chi=\phi$. Thus every minimizer in $\mathcal{E}(\Omega, A)$ lies in $\mathcal{D}(\Omega, A)$.

The theorem is proved, but another consequence for the minimizing sequence $\left(u_{n}, \chi_{n}\right)$ constructed in the proof is worth noting: We have just seen that $\phi:=$ $\mathrm{w}-\lim \chi_{n}$ is actually a characteristic function itself. Now invoke the simple

Lemma 1. Take any $1 \leq p<\infty$. Assume a sequence of characteristic functions $\chi_{n}$ on a finite measure space $X$ converges weakly in $L^{2}(X)$ to some function $\phi$. Then $\phi$ is a characteristic function if and only if $\chi_{n} \rightarrow \phi$ strongly in $L^{p}(X)$.

So we actually get $L^{2}$-strong convergence $\chi_{n} \rightarrow \chi$ for the minimizing sequence.

The proof of the lemma is as follows: If $\phi^{2}=\phi$, we get

$$
\begin{gathered}
\left\|\left(\chi_{n}-1\right) \phi\right\|_{L^{1}}=\int \phi\left(1-\chi_{n}\right)=\int \phi\left(\phi-\chi_{n}\right) \rightarrow 0, \\
\left\|\chi_{n}(1-\phi)\right\|_{L^{1}}=\int \chi_{n}(1-\phi)=\int\left(\chi_{n}-\phi\right)(1-\phi) \rightarrow 0,
\end{gathered}
$$


and therefore $\chi_{n}-\phi=\chi_{n}(1-\phi)+\left(\chi_{n}-1\right) \phi \rightarrow 0$ strongly in $L^{1}$. Using $\left\|\chi_{n}-\phi\right\|_{L^{\infty}} \leq$ 1 and the Hölder inequality, the same conclusion follows for $L^{p}$-convergence.

On the other hand, if $\chi_{n} \rightarrow \phi$ strongly in $L^{2}$, we conclude $\int\left(\phi-\phi^{2}\right)=0$, and with $\phi-\phi^{2} \geq 0$, this implies $\phi$ is a characteristic function.

It is a noteworthy feature of theorem 1 that, contrary to common practice, $D$ is not assumed to be closed, not even $\stackrel{\circ}{\bar{D}}=\stackrel{\circ}{D}$. Stampacchia ([17], thm 4.1) has shown minimizers (critical points) $u$ to be continuous up to the boundary under reasonably weak assumptions on the fixed closed set $D$. Whenever such a continuity result holds, the set $\{u=0\}$ will be closed automatically. However, to the author's knowledge, continuity up to the boundary has not been proved anywhere under the general assumptions on $D$ made here, most likely it will not even be true. It seems a reasonable conjecture, however, that optimal windows have a nice geometry and eigenfunctions continuous up to the boundary.

The strict monotonicity of $\ell$ prevents minimizing sequences from smearing out (i.e, converging weakly without converging strongly). Conversely, maximizing sequences typically exhibit weak convergence that is not strong. This behaviour is responsible for the fact that $\sup \left\{\lambda_{1}(D) \mid \mu(D)=A\right\}=\lambda_{1}(\partial \Omega)$, an easy proof of which has been given in [4]. Indeed, it was the sudden increase in effective window area for sequences $D_{n}$ exhibiting the smearing out phenomenon, which motivated the key idea of the proof, namely that broadening the class $\mathcal{D}(\Omega, A)$ to $\mathcal{E}(\Omega, A)$ should not change the problem.

Another observation is that minimization over the intermediate set with the constraints $1 \geq \phi \geq 0, \int_{\partial \Omega} \phi=A$ would have given the same result again. The characteristic functions $\chi$ are the extremal points of that subset of $L^{\infty}(\partial \Omega)$, so theorem 1 is of the type "minima are taken on only on extremal points of the domain of definition". This suggests that some yet undiscovered convexity structure may be behind the problem. It should be pointed out that a convexity argument does enter into the existence theorem for minimizers of the intermediate variational problem used in [4] to establish quantitative lower bounds for $\lambda_{1}(D)$.

\section{The Optimal Window in a Ball}

No results on how the (an) optimal window will look has been given in section 1 . Heuristic arguments (as given in [4]) suggest that it should look "round" rather than like a slit and probably be connected. In the special case of a ball, this can be made rigorous. The argument supplies an independent existence proof along with the explicit shape of the optimal window.

Theorem 3. Let $\Omega$ be a d-dimensional (unit) ball and $A<\mu(\partial \Omega)$. Then $D$ is an optimal window, i.e. $\lambda_{1}(D)=\ell(\mu(D)$ ), if and only if $D$ is a spherical cap (up to a null set).

The proof is by spherical symmetrization: Given a measurable set $K \subset \mathbb{R}^{d}$, the distinguished origin $0 \in \mathbb{R}^{d}$, and a distinguished direction (defining the north pole on all spheres centered at 0 ), the spherical symmetrization $K^{*}$ of $K$ is constructed as follows: For each $r$, take $K \cap \partial B_{r}(0)$ and replace it by the spherical cap of the same area and centered at the north pole of $\partial B_{r}(0)$. This can be done for a.e. $r$. The union of these caps is $K^{*}$. The spherical symmetrization $u^{*}$ of a measurable function $u \geq 0$ is constructed by symmetrizing the superlevel sets: $\forall t:\{u \geq t\}^{*}=\left\{u^{*} \geq t\right\}$. See e.g. [16] for more details. 
Symmetrization arguments have first been used by Steiner and Schwarz in the isoperimetric problem and then by Faber and Krahn in order to show that for a given area the minimal first Dirichlet eigenvalue is taken on for a disk. The classical reference for these types of results is [11].

Similar to Steiner and Schwarz symmetrization, one has the following

Theorem 4. For $u \in C^{\infty}\left(B_{r}(0)\right), u \geq 0$, it holds

$$
\int_{B_{r}(0)}\left|\nabla u^{*}\right|^{2} \leq \int_{B_{r}(0)}|\nabla u|^{2}, \quad \int_{B_{r}(0)}\left|u^{*}\right|^{2}=\int_{B_{r}(0)}|u|^{2} .
$$

For equality to hold in the gradient estimate, it is necessary that on each sphere $S_{r}(0)$, $u^{*}$ and $u$ coincide up to a rotation (which may depend on $r$ ).

The estimate, actually in more generality, is due to Sperner [16]. He does not discuss equality, and we give a proof of that part below. It is definitely not true that $\int_{B_{r}(0)}\left|\nabla u^{*}\right|^{2}=\int_{B_{r}(0)}|\nabla u|^{2}$ would imply $u \circ R=u^{*}$ for some rotation $R$ : e.g, if $u$ happens to be constant on some sphere $S_{r}(0)$, a counterexample can be given by taking different rotations inside and outside that sphere.

Sperner uses this theorem for a different variational problem, where the symmetrization changes (and optimizes) $\Omega$. However, in our setting it is crucial to start with a ball $\Omega$ so that the symmetrization only changes the window, but not the room $\Omega$.

Choosing the lowest eigenfunction $u \geq 0$ for any given window $D, u^{*}$ will be a candidate for (0.1) with window $D^{*}$, a spherical cap, and hence $\lambda_{1}\left(D^{*}\right) \leq \lambda_{1}(D)$. Hence spherical caps are optimal windows.

We now discuss the case of equality in order to show that only spherical caps can be optimal windows. Let $n:=x /|x|$ be the radial unit vector and decompose $\nabla u=n\langle n, \nabla u\rangle+\nabla_{t} u$. It is an immediate corollary (functions independent of the radial variable) of theorem 4 or else it can be shown independently by more or less the same method [15] that for functions $u$ on a sphere $S_{r}(0)$,

$$
\int_{S_{r}(0)}\left|\nabla_{t} u^{*}\right|^{2} \leq \int_{S_{r}(0)}\left|\nabla_{t} u\right|^{2}
$$

holds. On the other hand, we will argue that

$$
\int_{S_{r}(0)}\left\langle n, \nabla u^{*}\right\rangle^{2} \leq \int_{S_{r}(0)}\langle n, \nabla u\rangle^{2} .
$$

(Clearly, (2.2), unlike (2.1), depends on the values of $u$ in a neighbourhood of the sphere.) If equality holds in theorem 4, it holds in both (2.1) and (2.2) for almost every $r$. Going through the proof of (2.1) in [15], and denoting by $\sigma$ the $(d-2)-$ dimensional Hausdorff measure on the $(d-1)$-sphere, $\int_{S_{r}(0)}\left|\nabla_{t} u^{*}\right|^{2}=\int_{S_{r}(0)}\left|\nabla_{t} u\right|^{2}$ implies

$$
\begin{aligned}
\int_{S_{r}(0)}\left|\nabla_{t} u^{*}\right| & =\int_{0}^{\infty} \sigma\left(\left(\left.u^{*}\right|_{S_{r}(0)}\right)^{-1}\{z\}\right) d z=\int_{0}^{\infty} \sigma\left(\left(\left.u\right|_{S_{r}(0)}\right)^{-1}\{z\}\right) d z \\
& =\int_{S_{r}(0)}\left|\nabla_{t} u\right|
\end{aligned}
$$

and

$$
\left|\nabla_{t} u(x)\right|=f\left(\left.u\right|_{S_{r}(0)}(x)\right)
$$


for some function $f$ which may depend on $r$, whereas

$$
\begin{aligned}
& \sigma\left(\left(\left.u^{*}\right|_{S_{r}(0)}\right)^{-1}\{z\}\right)=\sigma\left(\partial\left(\left.u^{*}\right|_{S_{r}(0)}\right)^{-1}[z, \infty[)\right. \\
& \quad \leq \sigma\left(\partial ( u | _ { S _ { r } ( 0 ) } ) ^ { - 1 } \left[z, \infty[)=\sigma\left(\left(\left.u\right|_{S_{r}(0)}\right)^{-1}\{z\}\right)\right.\right.
\end{aligned}
$$

for a.e. $z$. But the middle inequality of (2.5) is just the isoperimetric inequality on the $(d-1)$-dimensional sphere, and it must become an equality in the present situation. So, $\left(\left.u\right|_{S_{r}(0)}\right)^{-1}[z, \infty[$ must be a disk (i.e. spherical cap) first for a.e. and then for every $z$. The sharp isoperimetric inequality on the sphere is discussed in $\left[13, \mathrm{II}\right.$, p. 231]. Then (2.4) implies that the caps are concentric, i.e., $\left.u\right|_{S_{r}(0)}=$ $\left.u^{*}\right|_{S_{r}(0)} \circ R_{r}$ for some rotations $R_{r}$.

We still need to justify (2.2): Expressing the derivative as a limit of difference quotients, this is an immediate consequence of $\int_{S_{r}(0)} u^{*} v^{*} \geq \int_{S_{r}(0)} u v$. This latter result can actually be seen as a result about Schwarz symmetrization in $\mathbb{R}^{d-1}$, using a measure preserving variant of the stereographic projection: the radial variable $\rho$ in $\mathbb{R}^{d-1}$ relates to the polar distance $\vartheta$ on the sphere by $\rho^{d-2} d \rho=(r \sin \vartheta)^{d-2} d \vartheta$. It is a simple special case of the higher dimensional version of Riesz's inequality [12] $\iint u^{*}(x) v^{*}(y) k^{*}(x-y) d x d y \geq \iint u(x) v(y) k(x-y) d x d y$. Let $k=k^{*}$ converge to a Dirac distribution for that purpose. The higher dimensional version was introduced by Sobolev [14], but without discussion of the convergence of an infinite sequence of symmetrizations used in the inductive step along the dimension. A complete and concise proof of an even more general result can be found in [3].

This finishes the proof of theorem 4.

We now finish the uniqueness part of theorem 3 . Let $u \geq 0$ be the eigenfunction for an optimal window $D$. For this $u$, equality holds in theorem 4 . $u$ satisfies $-\Delta u=\lambda u$ in $B_{1}(0)$ and is real analytic there. The restriction $u_{r}$ of $u$ to the sphere $S_{r}(0)$ of radius $r$ will be a monotonic nonincreasing function of the distance (on the sphere) from some $x_{r}$ alone, according to theorem 4 . (One can conclude strict monotonicity from analyticity and the fact that the Dirichlet problem for $\Delta+\lambda$ is uniquely solvable in $B_{r}(0)$, but we do not need this.) Now, as $r \rightarrow 1$, $u_{r}$ will converge to the trace of $u$ on the boundary in the strong $L^{2}$ sense (by compactness of the trace map). On the other hand, by compactness, $x_{r}$ will converge to some $x_{1}$ as $r \rightarrow 1$ on an appropriate subsequence. By Helly's theorem on monotonic functions, the $u_{r}$ will converge a.e. to some function $u_{1}$, which is a nonincreasing function of the distance from $x_{1}$ alone. The $L^{2}$ and a.e. limits must coincide, therefore the trace of $u$ has the mentioned monotonicity property. So it vanishes exactly on some spherical cap of $\partial B_{1}(0)$ whose measure equals $\mu\left(D^{*}\right)$. $D$ is a subset of this cap, but $\mu(D)=\mu\left(D^{*}\right)$. Therefore $D=D^{*}$ up to rotation.

The result as well as the method of proof suggest some relation between the shape of optimal windows and isoperimetrical problems. However, it is not clear yet how such a connection could be formulated for general rooms in detail.

Also note that for any set $D$ that is sandwiched between spherical caps $C_{-}$and $C_{+}$of radii $r-\varepsilon$ and $r+\varepsilon$ respectively, it will be true that $\lambda_{1}\left(C_{-}\right) \leq \lambda_{1}(D) \leq$ $\lambda_{1}\left(C_{+}\right)$, in particular, $\lambda_{1}(D)$ will be arbitrarily close to $\lambda_{1}(C)=\ell(\operatorname{vol}(C))$ (with $C$ the cap of radius $r)$, provided $\varepsilon$ is small enough. But such sets $D$ with $\mu(D)=\mu(C)$ exist with arbitrarily large perimeter. In other words, nearly optimal windows from the heat leak point of view need not be nearly optimal in the isoperimetric sense.

Similarly, given any $x_{0} \in \partial \Omega$ and any $\delta$, one can find an extra window of sufficiently small but positive area and located near $x_{0}$ that doesn't increase $\lambda_{1}$ by 
more than $\delta$. In particular, also the diameter of nearly optimal windows cannot be estimated in any nontrivial way.

Therefore any possible result along these lines cannot be a result on quasiminimizers, but must be a result on minimizers, and some version of Euler equations should be employed. At present, even a differentiable structure on a reasonable space of windows is lacking.

\section{Results on (Low) Elliptic Boundary Regularity for Mixed Conditions and Lipschitz Boundary}

In this section, we give some technicalities of elliptical regularity theory needed in the proof of theorem 1. The "hard" theorems are proved in the referred literature, but a detailed reference to the single ingredients and how they combine seems appropriate to make the proof clearly reproducible.

First we give some details of the proof that the minimizer $u$ of (0.1) is Hölder up to the boundary near points which are away from $D$. Due to the possibly bad geometry of $D$, we need to point out one subtlety not explicitly discussed in the standard references. Otherwise, the result is well-known.

The first step (following section 3.13 of [10]) is to use the weak Euler equations

$$
\int_{\Omega} \nabla u \cdot \nabla \phi d x=\lambda \int_{\Omega} u \phi d x \quad\left(\forall \phi \in H^{1}(\Omega) \text { such that }\left.\phi\right|_{D}=0\right)
$$

with the function $\phi:=\zeta^{2}(u-k)_{+}, \zeta$ a smooth cutoff function with support in $B_{\rho}\left(x_{0}\right)$, and $k \geq 0, x_{0} \in \Omega$ fixed (this does satisfy the boundary conditions). We let $\Omega_{\rho}:=\Omega \cap B_{\rho}\left(x_{0}\right), A_{k, \rho}:=\left\{x \in \Omega_{\rho} \mid u(x) \geq k\right\}$. As explained in [10], this implies

$$
\int_{A_{k, \rho^{\prime}}}|\nabla u|^{2} \leq \frac{c}{\left(\rho-\rho^{\prime}\right)^{2}} \int_{A_{k, \rho}}(u-k)_{+}^{2}+c k^{2} \operatorname{vol}\left(A_{k, \rho}\right)
$$

for $\rho^{\prime}<\rho$, where $c$ depends only on $\Omega$ and we have used the trivial upper bound for $\lambda$, namely the first Dirichlet eigenvalue. This inequality implies an upper bound for $u$ (cf. 2.(5.12) in [10]). The lower bound $u \geq 0$ happens to be trivial in our case. It is crucial that no geometric assumption on $D$ enters into the $L^{\infty}$ estimate. However, without such a geometric assumption, the next step, namely Hölder estimates up to the boundary cannot be carried through. This difficulty is natural, because for Dirichlet conditions, Hölderness of the boundary data must be used in the proof, whereas for Neumann conditions, the equation itself must give the result. If both types of conditions accumulate in one point, neither the proof for Dirichlet nor the one for Neumann conditions applies there.

A careful local discussion which, under moderate assumptions on the geometry of $D$, also works near the interface, where $D$ and $\partial \Omega \backslash D$ meet, can be found in [17]. However, in open subsets of the boundary that carry pure Neumann or pure Dirichlet conditions, the simpler thm 2.7.2 of [10] applies already, as discussed in section 3.14 there. But in any case the global $L^{\infty}$ estimate from the first step enters, and we could not have obtained it from local information alone.

This already proves the first part of the following lemma, the second part of which needs more recent techniques. 
Lemma 2. Any minimizer u for (0.1) for whatever measurable set $D$ is bounded in $\Omega$ and Hölder continuous up to any part of the boundary that has positive distance from either $D$ or $\partial \Omega \backslash D$. For almost every point $x_{0} \in \partial \Omega \backslash D$, one has $\left\langle\nabla u(x), \vec{n}\left(x_{0}\right)\right\rangle \rightarrow 0$ as $x \rightarrow x_{0}$ nontangentially.

We now need to (give a meaning to and) establish the claim that $\partial_{n} u=0$ on $(\partial \Omega \backslash D)^{\circ}$. A priori, $u \in H^{1}(\Omega)$, so its gradient does not have a trace on the boundary; on the other hand, $u$ will not be in $H^{2}(\Omega)$, if the domain has re-entrant corners.

We say that a function $u$ defined in the ball $B_{1}(0)$ converges nontangentially almost everywhere (ntae), if for every $\delta>0$ and a.e. $x_{0} \in \partial B_{1}(0)$, the limit $\lim _{x \rightarrow x_{0}} u(x)$ exists under the constraint that $\left\langle x-x_{0}, x\right\rangle /|x|\left|x-x_{0}\right|>\delta$. In this case, the limit will not depend on $\delta$.

In the case of a Lipschitz domain $\Omega$, neighbourhoods of sufficiently small parts of $\partial \Omega$ can be mapped into neighbourhoods of parts of the boundary of a unit ball by a bilipschitzian mapping. This defines convergence ntae in any Lipschitz domain (independent of the particular choice of the bilipschitzian mapping).

In order to localize near any compact subset $N_{0}$ of $(\partial \Omega \backslash D)^{\circ}$, let $\varphi \geq 0$ be a $C^{2}$ function that is identically 1 in a neighbourhood of $N_{0}$, with support disjoint from $\bar{D}$, and define $\tilde{u}:=u \varphi$. Then we have $\Delta \tilde{u}=-\tilde{f}, \partial_{n} \tilde{u}=\tilde{\psi}$ in the variational sense, i.e.:

$$
\int_{\Omega} \nabla \tilde{u} \nabla \phi=\int_{\Omega} \tilde{f} \phi+\int_{\partial \Omega} \tilde{\psi} \phi \quad\left(\forall \phi \in H^{1}(\Omega)\right)
$$

with

$$
\tilde{f}=\lambda u \varphi-2 \nabla u \cdot \nabla \varphi-u \Delta \varphi \in L^{2}(\Omega) \hookrightarrow L^{2}\left(\mathbb{R}^{d}\right), \quad \tilde{\psi}=u\left(\partial_{n} \varphi\right) \in L^{\infty}(\partial \Omega),
$$

where we have used the boundedness of $u$ (on the support of $\varphi$ ). $\tilde{f}$ is defined to vanish outside $\Omega$. $\tilde{u}$ satisfies a pure Neumann boundary problem on all of $\Omega$. The outside normal vector $\vec{n}$ implicit in $\partial_{n}$ is defined a.e. on $\partial \Omega$.

We let $\bar{u}(x):=-(\Gamma * \tilde{f})(x):=-\int_{\mathbb{R}^{d}} \Gamma(x-y) \tilde{f}(y) d y$ where $\Gamma$ is the fundamental solution $[6,(2.12)]$ in $\mathbb{R}^{d}$. Then, $\Delta \bar{u}=-\tilde{f}$ and, according to the Calderón-Zygmund estimates [6, thm. 9.9], $\bar{u} \in H^{2}(\Omega)$. As such, its gradient has a trace in $H^{1 / 2}(\partial \Omega)$, in particular $\partial_{n} \bar{u} \in L^{2}(\partial \Omega)$, and we have

$$
\int_{\Omega} \nabla \bar{u} \nabla \phi=\int_{\Omega} \tilde{f} \phi+\int_{\partial \Omega}\left(\partial_{n} \bar{u}\right) \phi \quad\left(\forall \phi \in H^{1}(\Omega)\right) .
$$

Actually, near $N_{0}$ (i.e., where $\varphi \equiv 1$ ), $\tilde{f}$ is in $L^{p}$ for all $p \leq \infty$, and therefore $\bar{u}$ will be a $W^{2, p}$ function there, for all $p<\infty$, due to the Calderón-Zygmund estimate again. By Sobolev's imbedding, $\nabla \bar{u} \in C^{\beta}$ in that neighbourhood of $N_{0}$ for all $\beta<1$. In particular, $\nabla \bar{u}$ converges uniformly to its boundary values, and therefore $\left\langle\nabla \bar{u}(x), \vec{n}\left(x_{0}\right)\right\rangle \rightarrow\left\langle\nabla \bar{u}\left(x_{0}\right), \vec{n}\left(x_{0}\right)\right\rangle=: \partial_{n} \bar{u}\left(x_{0}\right)$ as $x \rightarrow x_{0}$ for a.e. $x_{0}$.

Because of (3.3), (3.4), $h:=\tilde{u}-\bar{u}$ is a harmonic function with Neumann boundary data $\psi:=\tilde{\psi}-\left(\partial_{n} \bar{u}\right) \in L^{2}(\partial \Omega)$. In particular, $\int_{\partial \Omega} \psi=0$. For the Laplacian in Lipschitz domains, this implies that for a.e. $x_{0},\left\langle\nabla h(x), \vec{n}\left(x_{0}\right)\right\rangle$ converges to $\psi\left(x_{0}\right)$ as $x \rightarrow x_{0}$ nontangentially. This is the content of cor. 2.1.11 combined with remark 2.1.18 in [8]. 
Hence $\left\langle\nabla \tilde{u}, \vec{n}\left(x_{0}\right)\right\rangle$ converges (in the same sense) to its boundary values $\tilde{\psi}\left(x_{0}\right)$, which vanish on $N$. Using $\tilde{u} \equiv u$ near $N$, we have therefore completed the proof of lemma 2.

Proof of theorem 2. This proof is due to Kenig. I owe it to private communication by J. Pipher and him. The result is closely related to the unique continuation problem. However, there is no published reference for the theorem in the version needed here, so I detail out the proof, also attempting to make the ideas easily accessible to non-specialists. References to the original papers that coalesced into a theory for divergence form operators with nonsmooth coefficients or in nonsmooth domains can be found in Kenig's recent book [8], which collects these results. As a recent culmination, we mention [9]; the (smooth) Laplacian on Lipschitz domains is somewhat simpler and admits stronger results. It has been treated in [7].

In a first step we prove the theorem under the extra assumptions that $\Delta u \equiv 0$ and $u$ vanishes on an open (in $\partial \Omega$ ) set $\tilde{\Sigma} \supset \Sigma$. In this case, for any other nonnegative harmonic function $G$ vanishing on $\tilde{\Sigma}$, the comparison principle [8, lemma 1.3.7] will imply that there exists a constant $c$ and a neighbourhood $T$ of $\tilde{\Sigma}$ in $\bar{\Omega}$ such that $G \leq c u$ there, unless $u$ vanishes somewhere in $T^{\circ}$ and hence identically. Then, for $x_{0} \in \Sigma$,

$$
0 \leq \frac{G(x)-G\left(x_{0}\right)}{\left|x-x_{0}\right|} \leq c \frac{u(x)-u\left(x_{0}\right)}{\left|x-x_{0}\right|} \rightarrow 0
$$

as $x \rightarrow x_{0}$ "radially", i.e. $x-x_{0} \| \vec{n}\left(x_{0}\right)$. We conclude $\partial_{n} G\left(x_{0}\right)=0$. The expressions in (3.5) make sense as $u, G$ will be Hölder up to $\tilde{\Sigma}$ in that case. The argument holds in particular for Green's function $G$ [8, thm. 1.2.8]. However, the normal derivative of Green's function is the density of the harmonic measure with respect to the Lebesgue measure, and in the case of a Lipschitz domain, the harmonic measure cannot vanish on a set of positive Lebesgue measure, because both are absolutely continuous with respect to each other [8, thm 2.1.5]. (The classical proof by Green's integral formula carries over to $\partial_{n} G$ for Lipschitz boundary, if we test the harmonic measure on sufficiently regular boundary data and use section 2.1 of [8].)

This proves the first step, and next we drop the assumption that $u$ vanishes in $\tilde{\Sigma} \supset \Sigma$. Assume only that it vanishes in the set $\Sigma$ of positive measure (but still $\Delta u \equiv 0)$. Take open sets $\tilde{\Sigma}_{k} \supset \Sigma$ and let $u_{k}$ be the harmonic function with boundary values 0 on $\tilde{\Sigma}_{k}$ and $u$ on $\partial \Omega \backslash \tilde{\Sigma}_{k}$. These boundary data are in $L^{2}(\partial \Omega)$, and the existence and uniqueness of $u_{k}$ is guaranteed by thm 1.7.7 and cor. 2.1.6 of [8]. As $0 \leq u_{k} \leq u$ from the maximum principle, the same argument that showed $\partial_{n} G=0$ above now shows $\partial_{n} u_{k}=0$ on $\Sigma$. Therefore, by the first step, $u_{k} \equiv 0$. This time, $u\left(x_{0}\right)=0$ is taken on in the sense of convergence ntae, which is still good enough for (3.5) to apply a.e.

Now, we can take $\tilde{\Sigma}_{k}$ such that $\left\|u_{k}-u\right\|_{L^{2}(\partial \Omega)} \rightarrow 0$, and this implies (see 1.7.3\&7 of [8]) $\left\|u_{k}^{*}-u^{*}\right\|_{L^{2}(\partial \Omega)} \rightarrow 0$, where $u^{*}, u_{k}^{*}$ is the nontangential maximal function [8, p. 13]. (Essentially, $u^{*}\left(x_{0}\right):=\sup \left\{|u(x)|: x \in\right.$ an inner cone at $\left.x_{0}\right\}$.) Therefore $u^{*} \equiv 0$ and hence $u \equiv 0$.

In the last step we treat the general case. Denoting by $h$ the harmonic function with the same boundary values as $u$, we get $0 \leq h \leq u$, and $h=0, \partial_{n} h=0$ on $\Sigma$. Hence $h \equiv 0$ as before. So we are left with $\Delta u=: f \leq 0$ where $u$ vanishes on the boundary $\left(u \in \stackrel{\circ}{H}^{1}(\Omega)\right)$. This implies $u(x)=\int_{\Omega} G(\bar{x}, y) f(y) d y$ with $G$ being 
Green's function again. For any compact $K \subset \subset \Omega$, let $u^{[K]}(x):=\int_{K} G(x, y) f(y) d y$. From $0 \leq u^{[K]} \leq u$, we get $\partial_{n} u^{[K]}=0$ wherever $\partial_{n} u=0$. But $u^{[K]}$ is harmonic near $\partial \Omega$ (namely in $\Omega \backslash K$ ), so $u^{[K]} \equiv 0$ there. But now, due to the strong maximum principle for superharmonic functions $\left[6\right.$, Thm. 3.5], this means that $u^{[K]} \equiv 0$ in all of $\Omega$. Letting $K \nearrow \Omega$, we get $u^{[K]} \nearrow u$ and therefore $u \equiv 0$.

\section{ACKNOWLEDGEMENTS}

The existence result would have remained with unnaturally strong regularity assumptions without Carlos Kenig supplying me with theorem 2. I'd like to express my gratitude for this contribution. I'd also like to thank Jill Pipher for communicating this contact and for many valuable discussions on elliptic theory in general, which improved my insight. I also profited from discussions on symmetrization with Almut Burchard and Robert McCann.

Without Walter Craig's inventing the problem and the early discussions with him and the hospitality of the Lefshetz Center of Dynamical Systems at Brown University, which I enjoyed during the academic year 1994/95, this project could not have come into existence.

\section{REFERENCES}

1. R.A. Adams: Sobolev Spaces, Academic Press, 1978 MR 56:9247

2. S. Agmon, A. Douglis, L. Nirenberg: Estimates near the Boundary for Solutions of Elliptic Partial Differential Equations Satisfying General Boundary Conditions, Comm. Pure Appl. Math. 12 (1959), 623-727 MR 23:A2610

3. H.J. Brascamp, E.H. Lieb, J.M. Luttinger: A general rearrangement inequality for multiple integrals, Journal of Functional Analysis 17 (1974), 227-237 MR 49:10835

4. J. Denzler: Bounds for the heat diffusion through windows of given area, J. Math. Anal. Appl. 217 (1998), 405-422. CMP 98:07

5. H. Federer: Geometric Measure Theory, Springer 1969 (Grundlehren 153, now reprinted in the series Classics in Mathematics) MR 41:1976

6. D. Gilbarg, N.S. Trudinger: Elliptic Partial Differential Equations of Second Order, Springer Grundlehren 224 MR 57:13109

7. D.S. Jerison, C.E. Kenig: The Neumann problem on Lipschitz domains, Bull. AMS 4 (1981), 203-207 MR 84a:35064

8. C.E. Kenig: Harmonic Analysis Techniques for Second Order Elliptic Boundary Value Problems, CBMS, Vol. 83, American Mathematical Society, 1994 MR 96a:35040

9. C.E. Kenig, J. Pipher: The Neumann problem for elliptic equations with non-smooth coefficients, Inventiones mathematicae 113 (1993), 447-509 MR 95b:35046

10. O.A. Ladyzhenskaya, N.N. Ural'tseva: Linear and Quasilinear Elliptic Equations, Academic Press 1968 MR 39:5941

11. G. Pólya, G. Szegő: Isoperimetrical Inequalities in Mathematical Physics, Annals of Mathematics Studies 27, Princeton Univ. Press, 1951 MR 13:270d

12. F. Riesz: Sur une inégalité integrale, Journal of the London Mathematical Society 5 (1930), $162-168$

13. E. Schmidt: Die Brunn-Minkowskische Ungleichung und ihr Spiegelbild sowie die isoperimetrische Eigenschaft der Kugel in der euklidischen und nichteuklidischen Geometrie; part I: Mathematische Nachrichten 1 (1948), 81-157; part II: 2 (1949), 171-244. MR 10:471d; MR 11:5341

14. S. Sobolev: Ob odnor teoreme funktsional'nogo analiza (On a theorem of functional analysis), Matematicheskiı̌ Sbornik N.S. 4(46) (1938), 471-497

15. E. Sperner: Zur Symmetrisierung von Funktionen auf Sphären, Mathematische Zeitschrift 134 (1973), 317-327 MR 49:5310 
16. E. Sperner: Spherical Symmetrization and Eigenvalue estimates, Mathematische Zeitschrift 176 (1981), 75-86 MR 82e:35062

17. G. Stampacchia: Problemi al contorno ellittici, con dati discontinui, dotati di soluzioni hölderiane, Annali di Matematica Pura ed Applicata 51 (1960), 1-37 MR 23:A3897

Mathematisches Institut, Ludwig-Maximilians-Universität, Theresienstrasse 39, D80333 München, Germany

Current address: Zentrum Mathematik, Technische Universität, Arcisstrasse 21, D-80290 München, Germany

E-mail address: denzler@mathematik.tu-muenchen.de 